\title{
Reproducción en la revolución. Género y mujeres frente a la ley francesa de paridad en política ${ }^{1}$
}

\author{
Delphine DuLONG y Frédérique MATONTI
}

$\mathrm{E}$ 16 de junio de 2000, el gobierno socialista francés de Lionel Jospin sometió a votación una ley "que busca favorecer el igual acceso de las mujeres y de los hombres a los mandatos electorales y a las funciones electivas". Mucho más ambiciosa que los distintos sistemas de cuotas vigentes, esta ley estableció de manera inédita la paridad en la representación entre hombres y mujeres para todas las elecciones por lista. En las elecciones municipales (para las comunas de 3.500 y más habitantes), regionales, europeas y senatoriales (para los departamentos con 4 escaños y más), la ley obligó entonces a los partidos a colocar al mismo número de hombres y mujeres en los seis primeros lugares de las listas electorales. Para las elecciones parlamentarias, en las cuales se aplica un sistema electoral mayoritario con dos vueltas, la ley es sólo incentivadora: prevé sanciones financieras para los partidos que no inscriban igual número de mujeres y hombres.

Para las mujeres y los hombres que la presentaron ante el Parlamento (Lagrave, 2000; Bereni, 2004), esta affirmative action debía subsanar el "escandaloso" déficit de representación de las mujeres en las distintas

1. Este artículo presenta de manera sintética los principales resultados de una encuesta colectiva - “La invención de la representante elegida: estudio acerca del ingreso de las mujeres en política" - financiada por el Ministerio francés de Investigación (ACIJC $\mathrm{n}^{\circ}$ 67016). Esta encuesta fue realizada por 15 investigadores, quienes pertenecen a 4 laboratorios. Se aplicó en forma simultánea en 8 lugares distintos y durante un período de 5 años (2001-2005), de tal manera que se pudo observar más de seis elecciones locales y nacionales, y hacer un seguimiento de las mujeres elegidas en el ejercicio de su mandato. Se usaron métodos muy variados, pero se privilegiaron las metodologías cualitativas: observación de las campañas electorales y de varias instancias de poder (asambleas regionales, concejos municipales, secciones de partidos políticos), realización de más de 150 entrevistas, análisis de documentos de campaña (volantes y programas) y análisis de la prensa (generalista y femenina). Traducción de Emmanuelle Barozet. 
instancias del poder político en Francia, ayudar a que se tomaran en cuenta los intereses propios de las mujeres en las políticas públicas -y por lo tanto su condición social- pero también debía cambiar la vida política mediante la feminización de sus cargos. La revolución, sin embargo, no ha ocurrido. Basta con estudiar la composición estadística de los cargos políticos según el sexo para convencerse de ello. Por cierto, donde no se aplica, o donde es sólo incentivadora, la ley ha tenido muy poca adhesión. La proporción de mujeres elegidas en los Concejos generales $^{2}$ (donde la ley no se aplica) aumentó sólo en unos 3,5 puntos entre 1998 y el 2001 (pasando de 6,3\% a 9,8\%) y en 4,4\% en la Asamblea nacional, que cuenta con sólo un 12,3\% de diputadas, contra un 7,9\% anteriormente. Sobre todo, e incluso donde la paridad es obligatoria, los representantes de sexo masculino conservan el poder real, pues siguen monopolizando los puestos del Ejecutivo. En 2004, solamente un 3\% de los presidentes de Concejos generales eran mujeres, y si bien son más numerosas a la cabeza de las instancias municipales (pasando de 7,5\% a $11 \%$ ), éstas se encuentran netamente subrepresentadas en los órganos ejecutivos de las instancias intercomunales, que son las instituciones donde se expresa realmente el poder a nivel comunal (con un $0,3 \%$ entre los presidentes de los $\mathrm{ECPI}^{3}$ ). Finalmente, en algunos casos, la proporción de mujeres ha disminuido. Esta es la realidad, en particular, en los órganos ejecutivos regionales: si bien existen más vicepresidentas (un $37,3 \%$ contra un $15,1 \%$ anteriormente), una sola mujer se encuentra en la cabeza de una región ${ }^{4}$.

Por otro lado, en las asambleas paritarias, el modo de atribución de las tareas sigue obedeciendo a la división más tradicional de las tareas sexuales. Un estudio realizado en 85 comunas en 2001 (Libération, 31 de marzo del 2001) indica que el $65 \%$ de las mujeres elegidas en los Concejos municipales se han encargado de los dossiers de acción social, un $40 \%$ de los de cultura, un 35\% de medio ambiente; a contrario, sólo un $15 \%$ se dedica a los temas de finanzas, un $15 \%$ a los de seguridad y un $11 \%$ a los de transporte. Cabe agregar que esta repartición sexuada del trabajo político es igual en las otras asambleas paritarias regionales, puesto que los asuntos sociales, la vivienda y la protección social siguen siendo tareas ampliamente femeninas. Esta división del trabajo también se ve durante

2. N.d.T: los Concejos generales son asambleas que rigen el funcionamiento y las políticas de los 101 departamentos franceses. Sus miembros (cada uno representa un cantón) son elegidos cada 6 años (la mitad del Concejo se renueva cada 3 años) por sufragio universal uninominal.

3. Establecimiento Público de Cooperación Intercomunal. N.d.T.: Esta institución se encarga de la gestión de los servicios públicos, de la ordenación del territorio y del desarrollo económico entre comunas.

4. N.d.T.: Existen 22 regiones en Francia, las que, en virtud de la Ley de descentralización de 1982, cuentan con una asamblea (Concejo regional), elegida de la misma manera que los Concejos generales. Los Concejos regionales tienen atribuciones parecidas a los Concejos generales, pero a nivel regional. 
las sesiones plenarias de dichas asambleas paritarias. Un trabajo de observación llevado a cabo durante un período de un año en el Concejo regional d'île-de-France ${ }^{5}$ (Dulong, Matonti, 2005a) muestra efectivamente que las desigualdades persisten en el uso de la palabra en público. Cualquiera sea el tipo de intervención, los hombres siempre toman más la palabra que las mujeres. En 8 sesiones, los hombres intervinieron 142 veces y las mujeres 80 durante la discusión general. Dos tipos de intervención confirman aún más la performance de los hombres: las intervenciones relacionadas con la técnica parlamentaria ${ }^{6}$ y las intervenciones relacionadas con temas como los juegos olímpicos, la democracia regional y los transportes. La relación es de 7 para los hombres a 2 para las mujeres respecto a estos temas técnicos, que pueden ser considerados como "masculinos" desde un doble punto de vista, pues corresponden a supuestas cualidades "naturalmente" masculinas y porque son abordados sistemáticamente por hombres (Dunezat, 1998).

En resumen, cualquiera sea el indicador, todos los elementos convergen hacia una misma constatación: no solamente la revolución no ha ocurrido, sino que tiende a producir un campo político con un clivaje sexual, y también jerarquizado: para los hombres, las posiciones dominantes, y para las mujeres, las posiciones dominadas. Queda entonces por explicar este fenómeno de reproducción en la revolución. Veremos en un primer momento que los efectos que se esperaban de la reforma paritaria han sido ampliamente neutralizados por las lógicas específicas del campo político y, en un segundo momento, que ha chocado con los principios de la división sexuada del mundo social.

\section{La ley de paridad frente a las reglas tácitas del campo político}

Como lo han mostrado varios trabajos de sociología política, los campos políticos nacionales son por definición autónomos una vez estabilizados los regímenes políticos. El comportamiento de los individuos dentro de ellos está regulado en gran parte por reglas a la vez normativas y pragmáticas (Bailey, 1971), que no están inscritas en ninguna parte (sino en la cabeza de los actores), pero que no dejan de ser menos obligatorias. Por cierto, "todo jugador marginal o aspirante puede intentar modificar las reglas de funcionamiento del campo, sufre también su atracción por el mero hecho de ubicarse dentro de él" constata Michel Offerlé (2002: 91). Estas reglas son particularmente eficaces en Francia, país en el cual el campo político está muy cerrado frente a las lógicas sociales y especial-

5. N.d.T.: Región que agrupa los ocho departamentos de París y sus alrededores.

6. Las llamadas al reglamento siguen siendo intervenciones netamente masculinas (13 entre 16 provienen de hombres); sucede los mismo con las peticiones de explicación del voto, para las cuales hubo 51 intervenciones masculinas, contra 14 femeninas. 
mente frente a los movimientos sociales (Achin, 2005a). Por lo tanto, la paridad, a pesar de su fuerza legal, se enfrentó primero a varias de estas reglas específicas del mundo político.

\section{Elusión de la ley y autoexclusiones}

Si admitimos que la actividad política tiene como meta la conquista y la conservación del poder, no tenemos por qué asombrarnos de que los profesionales de la política hayan intentado limitar las consecuencias de la ley sobre su carrera. Toda la economía de las retribuciones del militantismo (Gaxie, 1997) se encuentra inicialmente cuestionada por la regla paritaria: además de volver más complejo el respeto por el equilibrio intrapartidista y por los equilibrios territoriales en cuanto a la elaboración de las listas electorales, disminuye mucho el número de puestos para los militantes masculinos, a pesar de que son mucho más numerosos que las mujeres en los partidos políticos ${ }^{7}$. ¿Cómo, entonces, desbancar a éstos? ¿Qué se puede hacer con los representantes salientes? Estas son las dos principales dificultades que las directivas de los partidos políticos enfrentaron, no sólo al incorporar mujeres, contrariamente a lo que algunos sostenían, sino por la obligación de encontrar respuesta a estos problemas que no son inocuos para la disciplina intrapartidista.

Las principales controversias durante la discusión constitucional hacían augurar este tipo de problemas. De hecho, una de sus consecuencias decisivas fue la limitación del carácter obligatorio de la ley, pues ésta se aplica solamente a las elecciones por lista, transformando la paridad en una ley de impulso, más que una regla obligatoria. Lo que quedó del proyecto inicial pudo entonces fácilmente ser esquivado durante su aplicación. Ya que la composición de los órganos ejecutivos -a fortiori de los gabinetes políticos- no estaba sometida a la regla paritaria, nada impedía a las cabezas de lista relegar a las mujeres a lugares subalternos después de la elección. Las estadísticas que evocamos indican qué fue lo que ocurrió, aunque se produzcan variaciones según las configuraciones políticas locales. Algunas cabezas de lista prefirieron rodearse de mujeres completamente novatas en política, particularmente cuando la victoria había sido difícil, para asentar su liderazgo sobre los órganos ejecutivos (Latté, 2002). Otros aprovecharon esta situación para deshacerse de colaboradores indeseados, fueran hombres o mujeres. En cuanto a los alcaldes reelegidos, éstos han sido los más reacios a aplicar la ley de paridad: ya que disponían de un menor margen de maniobra que los nuevos alcaldes respecto a la composición del ejecutivo municipal, la

7. En el 2003, según cifras entregadas por los partidos políticos, las mujeres representaban el $40 \%$ de los miembros del Partido Comunista, el 37\% de los miembros del partido gaullista Unión para un Movimiento Popular (UMP), el 35\% de los Verdes y el 34\% del Partido Socialista. 
regla pragmática de la postulación de los salientes jugó más bien en contra de la paridad (Troupel, 2002).

A este primer tipo de reglas del juego político, cabe añadir otras, que pesan igualmente sobre la elaboración de las listas y de los órganos ejecutivos. Consultado acerca de su voluntad de armar un Ejecutivo paritario en caso de triunfo, el presidente saliente de la región de Aquitania, afirmaba lo siguiente:

Ésta es la meta. Pero por supuesto, no se trata de entregar responsabilidad solamente por entregar responsabilidad, ¡no! Cabe asumirlas, por lo tanto cabe demostrar ciertas competencias (entrevista realizada el 18 de marzo del 2004 en Bayona).

El hecho de poseer una competencia, e incluso una "experticia" en un ámbito de acción pública, para poder aspirar al ejercicio del poder político, es una de las reglas tácitas más comunes en Francia desde el advenimiento de la V República (Dulong, 1997). Aunque no tiene nada que ver con la paridad, constituye también una traba grave a la igualdad de oportunidades entre hombres y mujeres, una traba aún más eficiente porque no ha sido orientada hacia este fin y que las mujeres, al someterse a ella, contribuyen a reforzarla de forma activa. Y de hecho, cualquiera sea el nivel político, las representantes afirman muchas veces haber solicitado las áreas que se les asignaron porque se sentían más "atraídas" por ellas, más "competentes" y más aptas para demostrar sus aptitudes. Porque desea tener una carrera política, esta nueva consejera regional de Île-de-France explica que no quiere:

[...] sobrecargarme, hacerme cargo de dossiers que no domino. Pensé, es mi primer mandato, tengo que llevarlo bien, con los medios que tengo [...]. Quería seguir con esta continuidad para estar a gusto, para integrarme conforme a mi mandato, para después orientarme hacia otra cosa. Y ¿por qué no?, perfeccionarme realmente en mi ámbito (Consejera regional socialista elegida en el 2004, entrevista realizada el 27 de mayo del 2004, París).

Por las mismas razones, son pocas las mujeres quienes piden trabajar en áreas "masculinas" como vivienda o transporte; y cuando lo hacen, ocure siempre que acumulan una experiencia profesional en estos ámbitos y un capital político (notoriedad, experiencia de representante, alta jerarquía en un partido, etc.) suficiente como para imponerse frente a los numerosos competidores atraídos por el prestigio de estas áreas. Entendemos mejor por qué el modo de atribución de las tareas en política sigue obedeciendo a la división sexual del trabajo: funciona por una parte como una prolongación de las actividades profesionales anteriores y obedece, por otro lado, a una lógica de cursus honorum, que reparte los puestos en función del capital político. Por lo tanto, las oportunidades de acceso de las mujeres a áreas tan prestigiosas como "masculinas" son más escasas que para los hombres. 
Sin embargo, la desnaturalización de lo que está inicialmente en juego con la reforma, se observa aún mejor con la autonomía del campo político. La reforma paritaria, como bien sabemos, nunca hubiera nacido sin la movilización de varios movimientos feministas. Para los hombres y mujeres que la promovieron en su inicio, el "escándalo" de la subrepresentación política de las mujeres en Europa justificaba este proyecto, subrepresentación que es parte de las desigualdades económicas y sociales persistentes entre hombres y mujeres (Bereni, 2004). Con todo, los desafíos propiamente feministas han desaparecido rápidamente de la escena política. Son pocas las candidatas y las representantes entrevistadas desde el 2001 que se reivindican feministas o que más simplemente defienden los derechos de las mujeres. Algunas, incluso, rechazan enérgicamente el calificativo "feminista", considerando "que es tan anticuado como el machismo", según comenta una teniente alcalde ${ }^{8}$ de una ciudad del suroeste de Francia. Sin embargo, este tipo de comportamiento nuevamente debe ser relacionado con las reglas del juego político francés, pues se trata de una forma de autocensura: cuando se observa a las representantes, sí muestran una verdadera conciencia de género y de las prácticas feministas.

En Francia, el feminismo no es considerado como una problemática política central por los profesionales de la política. Proclamarse "feminista" es muchas veces estigmatizante. De tal manera que la fuerte competencia inter e intrapartidista lleva a las candidatas y a las representantes que desean imponerse de forma perdurable a renunciar a la expresión perenne de una forma femenina de solidaridad ${ }^{9}$. Las representantes elegidas gracias a la reforma paritaria rápidamente han dejado de lado este punto, porque el "shock del 21 de abril del 2002"10 desvalorizó estos temas en beneficio de un interés por los temas de clase social, especialmente en la prensa. La exclusión en la segunda vuelta presidencial del candidato socialista que desafió al presidente Jacques Chirac, en prove-

8. N.d.T.: Después de la elección del alcalde por los concejales, éstos proceden a la elección de los tenientes alcaldes, quienes no pueden representar más del 30\% del número de concejales. Los tenientes alcaldes se encargan, de acuerdo con el alcalde, de asuntos específicos dentro de la comuna y son considerados como su brazo derecho.

9. De forma recíproca, gran parte de los movimientos feministas de la "segunda ola" (Chaperon, 2000) -la cual tuvo lugar en Francia en el periodo posterior a mayo del 68-, agrupados en el Movimiento de Liberación de las Mujeres, desconfiaron de la política institucional. En consecuencia, desplazaron sus luchas hacia la esfera social (lucha contra el patriarcado, para una sexualidad libre, para la contracepción, el aborto, etc.). Incluso, durante la actual tercera ola inaugurada en los años 90 (Achin, Lévêque, 2006), después del reflujo de los años 80, algunas feministas históricas radicales han lamentado que la instauración de la paridad obligue a entrar a la arena parlamentaria (Bereni, 2006).

10. N.d.T.: Ese día, en la primera vuelta de las elecciones presidenciales, los dos candidatos más votados resultaron ser Jacques Chirac, el Presidente saliente, con un 19,88\%, seguido por Jean-Marie Le Pen, el candidato del Frente Nacional, abiertamente xenófobo y racista, con un $16,86 \%$ de los votos, por lo cual Lionel Jospin, el candidato del Partido Socialista, quedó excluido de la segunda vuelta, con un $16,18 \%$ de los votos. 
cho del líder de la extrema derecha Jean-Marie Le Pen, fue percibida por la clase política (hombres y mujeres) como una conminación a volver a centrarse en objetivos políticos más tradicionales, privilegiando las lógicas partidistas nacionales y dejando poco espacio a la organización y la expresión de solidaridades categoriales (Lévêque, 2005).

\section{El género como recurso y como coacción en politica}

Sin embargo, este episodio coyuntural no lo explica todo. Primero, porque esta tendencia a la forclusión de las problemáticas femeninas se nota desde el momento en que se incluye la paridad en la agenda de las reformas gubernamentales. Luego, y en relación con lo anterior, porque estos temas han sido de golpe reemplazados por otros propiamente políticos (Dulong, 2003; Dulong, Matonti, 2005b). Desde que se iniciaron los debates sobre su adopción, la ley del 6 de junio del 2000 ha sido reintegrada dentro de los temas institucionales generales, los cuales no tenían nada que ver con el problema de la igualdad de oportunidades entre sexos. Como lo explica Lionel Jospin, entonces Primer Ministro, si bien la paridad política entre hombres y mujeres amerita una revisión constitucional, es "para que nuestra democracia, profundamente renovada por la feminización, tenga más dinamismo, vitalidad e imaginación"11. En el contexto de una "crisis" caracterizada por una fuerte abstención, por el aumento de los votos que apoyan partidos que no son partícipes de las coaliciones gubernamentales, por el declive del militantismo partidista y sindical, así como por frecuentes alternancias de las coaliciones en el poder (Matonti, 2005), la reforma paritaria ha sido de entrada concebida, presentada y adoptada como una manera de reencantar la vida política francesa. Y este punto, que fue pensado en un primer momento para caracterizar el campo político, se impuso a todos rápidamente, como se puede ver en la prensa o en las palabras de la directora del Observatorio de la Paridad entre hombres y mujeres, cuando explica en el diario Libération, apoyándose en los sondeos, que las mujeres:

[...] aportarían en las municipalidades más diálogo y concertación (83\%), tomarían más en cuenta las preocupaciones de los habitantes (81\%), aportarían más confianza entre las autoridades elegidas y la población $(60 \%)$. En otras palabras, esperamos que las mujeres reconcilien a los ciudadanos con la política (Claire Bernard, Libération, 31 de marzo-1 $1^{\circ}$ de abril del 2001).

Lejos de ser anecdótica, esta desnaturalización de lo que estaba en juego inicialmente en la reforma, pesó fuertemente al momento de su aplicación. Intimadas a humanizar la política -y a aterrizarla- en nombre de las cualidades imputadas a su género, las mujeres presentes en la competencia electoral, lógicamente, movilizaron el registro de la diferencia

11. Discurso ante la sesión plenaria del Parlamento, en Versalles, el 28 de junio de 2000. 
entre sexos (Dulong, Matonti, 2005b). Cualquiera sea su edad, profesión, comuna, años de experiencia militante o también su etiqueta política, las mujeres que se postularon a un mandato municipal en el 2001 se presentaron siempre como "más" o "menos" que los hombres: son "más disponibles", "más cercanas", "más concretas", "más responsables", "más conciliadoras", y a la inversa, "menos fanáticas", "menos ambiciosas", etc. Ahora bien, se trata de un fenómeno inédito en Francia, donde las mujeres, percibidas como ilegítimas en política porque son diferentes (Sineau, 1988), en general solían profesionalizarse en este sector de actividad, adoptando un comportamiento conformista (es decir, imitando los comportamientos "masculinos").

La primera consecuencia de este fenómeno consistió en posibilitar un esfuerzo (a la vez colectivo e individual) de constitución de una "identidad estratégica" (Collovald, 1999) femenina, mediante el cambio del estigma. Lejos de padecer su condición de género, las candidatas supieron jugar con ella durante las elecciones, muchas veces de manera pertinente, como lo afirma esta candidata en las elecciones legislativas de Lyon en el 2002:

Soy una mujer joven, juego con esto. Es una ventaja innegable si sabes usarla. En mi diario de campaña, había una foto enorme de mí en el medio (risas) -un verdadero afiche- ipero les desafío, cualquiera sea la circunscripción y el color político, a encontrar un diario electoral en el cual se habla tanto de política!" (Barbara Romagnan, Lyon Capitale, $n^{\circ} 384$, julio del 2002).

Cabe, sin embargo, matizar estas palabras, por lo menos por dos razones. La primera razón consiste en que si bien las mujeres no son totalmente víctimas de su género en política y saben jugar con él, no es cierto en cambio que puedan salirse de él. En efecto, las mujeres presentes en la competencia no han tenido una real libertad para elegir el registro en el cual juegan (Dulong, Matonti, 2005b). Esta presentación “femenina" de sí misma se les impuso como una constricción insuperable. La mejor prueba de ello es que incluso las más profesionales, las que disponen de un fuerte capital social y/o político, han sido intimadas a volver explícita su "femineidad". Vimos por ejemplo a Arlette Laguiller, líder de la organización trotskista Lucha Obrera, sacándose fotos en su cocina para la revista popular Paris-Match, cuando siempre había sido muy discreta respecto a su vida privada. También vimos a Martine Aubry, ex ministra socialista de Economía, en la portada de la misma revista, así como en la revista femenina Elle, explicando que aspiraba a la alcaldía de Lille para "juntarse con el hombre a quien ama". Este tipo de presentación de sí misma se inscribe por supuesto en la constitución de una identidad estratégica. Así lo explica uno de los consejeros en comunicación de Martine Aubry:

La estrategia consistía en decir, es una mujer, entre comillas, como las otras. Tiene a su pareja, sale de compras, está en la vida cotidiana, [...] es lo que dice en la revista Elle, trabajamos mucho está fórmula, "no soy una 
wonderwoman". Era la idea, en términos de comunicación, mostrar a las mujeres que es igual a ellas. Que tenía los mismos problemas, que tenía que cuidar a su hija, ella también, que tenía que salir de compras, que ella también tenía que... aunque obviamente no sea cierto... y que no era la mujer fuerte, inaccesible para las otras mujeres, que lograba llevar adelante una carrera de ministra, siendo número dos o tres del gobierno, criar a sus hijos (Entrevista realizada en París el 22 de octubre del 2001).

Aunque el artículo publicado en Paris-Match es el apogeo de una estrategia de comunicación, este mismo consejero explica de manera simultánea que estaban sometidos a fuertes presiones. En efecto, esta estrategia respondía a una serie de artículos "muy duros" acerca de Martine Aubry, donde entre otras cosas se "la tildaba de Elena, por Elena Ceaucescu", donde se decía que "esclaviza a sus colaboradores", que es "autoritaria, muy dura con toda la gente, que piensa que son todos tontos, etc...". Respondía también a un rumor acerca de la homosexualidad de Martine Aubry, difundida incluso en algunas federaciones del Partido Socialista.

Esto muestra hasta qué punto lo que por un lado aparece como un esfuerzo de construcción de una identidad estratégica femenina, se parece mucho por otro lado a un proceso de reclusión de las mujeres en su identidad sexuada. Existe una segunda razón que invita a matizar la idea según la cual las mujeres podrían instrumentalizar completamente su género en política. Los intentos de reconversión del género femenino en recurso político son en los hechos muy limitados y, para decirlo todo, muy controlados (Dulong, Lévêque, 2002). Primero, porque aparte del género, existen muchos otros recursos en política, como por ejemplo la profesión. Mientras más competitiva la situación y más partidistas los recursos, la primera tiende a primar sobre los segundos. Además, no se puede prescindir de estos recursos partidistas para hacer campaña, puesto que son los más colectivos y los más estrictamente políticos y militantes. De este modo, durante las elecciones parlamentarias y regionales, donde la competencia es doble -dentro del partido para imponer su candidatura y entre los candidatos de los diferentes partidos- ya no se trata de buscar candidatos, sino de desbancar varios de ellos. Lógicamente, los "vencedores" son los candidatos que tienen más recursos políticos, y el género ya no es un recurso suficiente para ser proclamado candidato.

Al respecto, el género no es solamente un recurso entre otros en política: es también un recurso más contingente que otros. De hecho, fue solamente durante las elecciones municipales de 2001 y en menor medida durante las elecciones regionales de 2004 que las mujeres lo hicieron explícito. No ocurrió nada parecido en las elecciones presidenciales y parlamentarias de 2002. El análisis cuantitativo de los programas electorales de los candidatos a diputado en París muestra que las estrategias de presentación de sí mismo, examinadas desde un punto de vista global, varían poco en función del género de los candidatos (Lévêque, 
2005). Las candidatas no parecen sobrecargar su propuesta con los atributos más "privados" de su identidad, como la familia o su modo de vida. A cambio, entre los recursos personales, valoran los atributos que dicen relación con su profesión. Si bien el "shock del 21 de abril del 2002" es por un lado un factor explicativo, como ya lo subrayamos, y que, por otro lado, los programas electorales sólo reflejan las condiciones objetivas de éxito (o de fracaso) en política ${ }^{12}$, otro factor explicativo son las propiedades socialmente construidas de las diversas competencias electorales en las cuales las mujeres están presentes. En el 2001, las mujeres postularon a las elecciones municipales, que corresponden al nivel más bajo en la jerarquía simbólica del poder político; en el marco de esta competencia local, los temas nacionales casi no se mencionaron ${ }^{13}$. Además, la coyuntura es bastante particular: todas las asociaciones de alcaldes se pusieron de acuerdo para centrar la atención de la población en el tema de la calidad de vida (Le Monde, 27 de enero de 2001) y la mayoría de las autoridades elegidas pensó que el ejercicio y la conquista del mandato municipal requería de ciertas cualidades relacionales consideradas como femeninas, tales como el "sentido del contacto humano", "la disponibilidad", "la capacidad de escucha" (Garraud, 1989). El registro de la "feminidad" fue entonces muy apropiado para esta campaña electoral. A la inversa, en el 2002, fueron otras las cualidades requeridas para asumir el papel de diputado (Achin, 2005b) - a fortiori de Presidente-. Como lo explica por ejemplo el ex ministro socialista de Cultura Jack Lang, durante un programa televisivo:

Un buen diputado no es una trabajadora social. Es alguien que debe estar cercano a los electores de su circunscripción, pero debe también saber conseguir de la administración central decisiones para su circunscripción y sobre todo debe ser un buen legislador. Debe tener entonces influencia a nivel nacional (Programa France Europe Express del 13 de enero de 2002).

Esto muestra que las mujeres pueden instrumentalizar su género con fines políticos solamente bajo ciertas condiciones, que además no controlan. De manera general, mientras más construida es la situación desde el punto de vista político (es decir con fuertes desafíos partidistas), más negativo es lo relacionado al género femenino. Por lo tanto, las mujeres pueden jugar menos con esto. Entonces, si bien hacer gala de los "atributos" del género femenino es posible e incluso rentable cuando la representación es dirigida hacia un público profano, es a cambio muy estigmatizante en lo interno (es decir dentro de los partidos políticos), donde es importante ser femenina, pero también es mejor no serlo demasiado.

12. En Francia, como en otras partes, sin lugar a duda, una mujer sola y sin hijos tiene más facilidades para hacer carrera en política que una mujer con hijo(s), aunque sea sólo por un tema de disponibilidad.

13. Prueba de ello es la desaparición de los símbolos partidistas en los programas de los candidatos. 


\section{Paridad y fortalecimiento de los estereotipos}

Pero si las mujeres, candidatas y representantes, han sido reducidas a sus identidades sexuadas, no es solamente porque han usado éstas estratégicamente y / o porque el juego político y todas sus reglas implícitas las han obligado a usarlas. Para entender esta reasignación identitaria, es necesario volver sobre la articulación entre orden social y orden político.

\section{La autonomía muy relativa de los campos políticos}

Si bien el campo político francés se caracteriza por un alto grado de autonomía respecto a las problemáticas sociales, esta clausura es, sin embargo, relativa, como lo comprueba por ejemplo, durante estos diez últimos años, la politización de las cuestiones sexuales (Fabre, Fassin, 2005) con las controversias públicas acerca del PACS ${ }^{14}$, de la pornografía, del matrimonio homosexual o del acoso sexual, y las consecuencias jurídicas de varios de estos temas. Por otro lado, los actores políticos están sometidos a los principios de clasificación del mundo social y, en este caso, a los principios que dictan cuáles son los espacios y los roles respectivos de los hombres y las mujeres, incluso solamente porque el acceso a las posiciones de poder depende de la movilización de apoyos fuera del campo político. Ahora bien, si las sociedades tradicionales, a través de sus mitos, proponen bajo una forma pura una "división de las cosas y de las actividades (sexuales u otras) en función de la oposición entre lo masculino y lo femenino" (Bourdieu, 1998: 13), las sociedades contemporáneas también siguen naturalizando y jerarquizando las oposiciones entre hombres y mujeres. De esta manera, mediante la educación y el trabajo de imposición realizados por instituciones como la familia, la Iglesia, el Estado o la escuela, las mujeres tienden a privilegiar la esfera privada, a elegir estudios literarios, a ejercer profesiones en continuidad con las tareas domésticas o el cuidado, a las cuales están "naturalmente" destinadas, etc. Y lo son aún más cuando, por una causalidad circular, la repartición de las posiciones sociales entre hombres y mujeres refuerza la creencia de cada uno de los actores de que las cosas son como deben ser. Es por esta razón que el ingreso de las mujeres en política pudo parecer (y parecerles a ella) como una trasgresión, aunque en numerosas sociedades diferenciadas, la dominación masculina ya no es obvia y se debe justificar cuando está presente. Se trata efectivamente de un espacio -la política- doblemente masculino o, más exactamente, pensado "naturalmente" como tal. Primero porque la presencia numérica de los hombres es abrumadora y luego porque todo lleva a creer que les está destinado.

14. N.d.T.: El Pacto Civil de Solidaridad es un contrato firmado entre dos personas adultas para organizar su vida común. Fue promulgado en 1999 con el fin de llenar el vació legal existente para las parejas homosexuales. 


\section{Las llamadas al orden de género}

El seguimiento de las campañas electorales y el examen de las asambleas elegidas muestran hasta qué punto el ingreso de las mujeres en política y su ascenso en este universo van de la mano con llamadas al orden de género del mundo social. La prensa es uno de los espacios por excelencia donde las mujeres son reducidas a esta identidad. Observamos cierto número de constantes ${ }^{15}$, tanto en los diarios de izquierda como de derecha, en la prensa generalista (quizá más aún) o en las revistas femeninas. El tono es siempre más familiar con las mujeres (muy a menudo llamadas por su nombre) que con los hombres. En los reportajes, su familia siempre está presente, a través de sus maridos, parejas, hijos, e incluso para las más jóvenes, sus padres. Pero tal vez lo más asombroso es la focalización de la prensa en el cuerpo -conformación, hexis y tratos intencionales (Bourdieu, 1977)- de las profesionales de la política. Por lo tanto, casi no existen artículos que no aludan a su porte, peso, aspecto, (nuevo) peinado, ropa..., incluso a su elegancia o apariencia desaliñada. Así, uno de los episodios del viaje a América Latina de la candidata socialista a la Presidencia de la República, Ségolène Royal, ha sido objeto de numerosos comentarios periodísticos acerca de sus tacos aguja, que se consideraron desubicados para visitar un barrio pobre. Igualmente, para la publicación de su libro, Marine Le Pen, quien podría ser la próxima presidenta del Frente Nacional, partido de extrema derecha dirigido por Jean-Marie Le Pen, su padre, es descrita de la manera siguiente:

Y con 11 kilos menos, su cara afilada y su falda plisada, la vicepresidenta del Frente Nacional desentona entre las fisionomías arrugadas y los peinados con permanentes azul eléctrico, reunidas este 15 de enero para comer el roscón de Reyes de la federación FN de París ${ }^{16}$.

A pesar de la ley de paridad, llama entonces la atención la inercia de las representaciones. Éstas son efectivamente comparables con las que Goffman, en su trabajo acerca de la publicidad y la manera en que refleja nuestros estereotipos, develó desde los años 70 (Goffman, 1988) o con las que Jane Freedman analizó más recientemente (Freedman, 1997).

Esta atención que la prensa presta a detalles no políticos no es solamente una manera inconsciente de recordar la identidad sexuada de las representantes. Es también el resultado de una "configuración de tres polos" (Neveu, 1997: 26): periodistas, personal político (incluidos los comunicantes) y opinión pública, tercer polo que es en gran medida "inventado" por los dos primeros. La focalización en el cuerpo de las mujeres

15. Parece, sin embargo (pero serían necesarios cálculos sistemáticos), que el diario comunista L'Humanité, siendo más atento que el resto de la prensa a las explicaciones sociales de los fenómenos políticos, recurre menos a estos estereotipos. Cabe también recordar que se trata del único partido francés hoy dirigido por una mujer, Marie-George Buffet.

16. Christophe Ono-Dit-Biot, "Marine de Guerre", Le Point, 13 de abril de 2006. 
políticas y en sus actividades supuestamente privadas, es el producto de una larga evolución y de lógicas profesionales de cada uno de estos universos. Tiene relación primero con un proceso multisecular de "desoficialización" de la representación de los cuerpos de los profesionales de la política (estamos lejos del retrato de los reyes "en majestad"). Luego, es el resultado de una modificación de la dramaturgia política, que estuvo durante mucho tiempo concentrada en los mitines y por consiguiente en un imaginario teatral (Cossart, 2001), pero más adelante sujetada por el medio dominante, la televisión. Ésta obliga a recurrir a un tono más íntimo, a una hexis más cotidiana, opuesta al tribuno, a la vez que invita a un encuadre estrecho de los cuerpos y de las caras. La evolución de la profesión periodística también lleva a mostrar los encuentros "entre bastidores", de manera de descartar las sospechas de connivencia entre periodistas y profesionales de la política (Legrave, 2002). Finalmente, en el marco de una "crisis de la representación políti$\mathrm{ca}^{\prime}$, se supone que las emociones y los gustos "revelados" de esta manera dicen la "verdad" acerca de la persona (Le Grignou, Neveu, 1983) y reducen la distancia entre profesionales de la política y profanos.

Resulta de lo anterior que la atención prestada al cuerpo puede también, y de manera marginal, afectar a los hombres políticos y parece reducir la brecha entre las percepciones estereotipadas acerca de hombres y mujeres. Así, el ex ministro socialista, Dominique Strauss-Khan, relató su dieta y su lifting de los párpados; el Presidente de la República, Jacques Chirac, escondió su sordera en contra de todas las evidencias; el Primer Ministro, Dominique de Villepin, toma un baño de mar, o sale a trotar con regularidad ante las cámaras, descubriendo un cuerpo tan deportivo que algunos humoristas e imitadores lo han representado con el cuerpo de una pin-up. Igualmente, las peripecias conyugales del ministro del Interior, Nicolas Sarkozy, dirigente de la UMP (Unión para un Movimiento Popular, partido de derecha) y candidato declarado a las presidenciales del 2007, dio mucho trabajo no solamente a la prensa popular, sino también a los periodistas políticos. Esto significa una doble ruptura con las tradiciones periodísticas francesas: hasta el momento no revelaban la vida sentimental de los políticos contra su voluntad, y por otro lado, cuando estos artículos hablan de las mujeres políticas, ponen un acento más marcado en la esfera privada.

Pero más allá de constatar la inercia general y algunas señales de evolución en este tema, lo que debe convocar nuestra atención son las llamadas al orden sexuado que esconde esta exhibición de estereotipos. Las representantes son reducidas a su papel tradicional de madres y esposas. Así, en la revista femenina Elle, cuyo feminismo puede ser calificado de reformista, la editorialista Ruth Elkrief realizó durante cerca de cinco años entrevistas cortas. Después de cinco o seis preguntas acerca de la parte más profesionalizada de la competencia política (elaboración de los programas políticos, de las listas electorales, competencia partidista 
interna en términos generacionales...), planteaba dos o tres preguntas sistemáticamente enfocadas a temas de la vida privada. Tanto en las preguntas como en las respuestas, cuando el entrevistado es un hombre, las parejas son reducidas a dos papeles principales: el papel de madre encargada de la educación de los hijos y el papel de esposa, atenta a la carrera de su compañero, benevolente, buena consejera, y eso gracias a sus cualidades emocionales y "femeninas" - pasión, precisión, paciencia, sensibilidad...- y no a sus competencias políticas. Estas mismas preguntas, planteadas a una mujer, las reduce a su papel de madre, jincluso cuando los hijos ya no viven con sus padres! Este es el ejemplo de Françoise de Panafieu, hoy candidata de la derecha a la alcaldía de París, a quien Ruth Elkrief hace la pregunta siguiente: "Tiene cuatro hijos, de los cuales tres estudian en el extranjero y la cuarta vive con ustedes en París. ¿Cuál es su reacción frente a su campaña electoral?". A la inversa, la periodista no le pregunta a Laurent Fabius, uno de los pocos hombres políticos con quien se habla de paternidad, lo que sus hijos piensan de las actividades de su padre, un tema que, sin embargo, fue detallado por el ex Primer Ministro en su autobiografía Las Heridas de la verdad ${ }^{17}$... A cambio, se le pregunta si sus hijos se dedicarán a la política.

Cabe añadir a esta reasignación de los roles más tradicionales una preocupación en la prensa por el tiempo libre y las actividades extraprofesionales de las mujeres políticas. Ahora bien, éstas no dudan en contestar y -por razones de comunicación, pero también por internalización de las normas- a hablar de las tareas "femeninas", es decir, las tareas realizadas por las mujeres y supuestamente afines a sus competencias. De esta manera, Élisabeth Guigou, entonces ministra de Justicia, en un reportaje realizado en su casa en Vaucluse, "coloca la loza en la lavadora" y cuida su jardín. Otras mujeres ministras hablan, de un reportaje a otro, de bordado, de costura, de lavadoras, etc. Esta interiorización de las normas sociales es tal, o más bien la suposición de que la "opinión pública" las tiene fuertemente interiorizadas es tan intensa, que estas mujeres políticas hablan de tareas domésticas de las cuales seguramente ninguna se encarga, debido a su estatus político y social.

Pero sin lugar a duda, el dato revelador es la manera en que se ejerce la llamada al orden sobre los cuerpos femeninos. Las numerosas autobiografías de mujeres políticas relatan como las campañas electorales o las sesiones en las asambleas siempre son espacios para chistes o comentarios sexistas, lo que confirma nuestras observaciones. Silbidos aparentemente admirativos cuando toman la palabra, toqueteos, rumores acerca de sus supuestas relaciones sexuales o acerca de su supuesta homosexualidad: las mujeres siempre son reducidas a su cuerpo. Más aún, este cuerpo es reducido al deseo que suscita (o que no suscita). Durante la III

17. Fabius (1998). 
República18, el ingreso de las mujeres a las profesiones "masculinas" (médico, abogado...) generó la misma reasignación a la alteridad sexual y sexualmente deseada: silbidos cuando las mujeres entraban en las aulas de las facultades de Medicina, postales y caricaturas con mujeres amamantando durante el alegato, etc. (Rennes, 2005). A final de cuentas, nada cambió respecto a lo que constataba Simone de Beauvoir:

Ella [la mujer] no es nada más que lo que el hombre decide que sea; por eso la llaman el "sexo débil", lo que quiere decir que se presenta (o aparece) ante el varón esencialmente como un ser sexuado para él, ella es sexo, por lo cual lo es de forma absoluta. Se determina y se diferencia respecto al hombre y no al revés; ella es lo inesencial frente a lo esencial. Él es el Sujeto. Ella es el Absoluto: ella es el Otro (De Beauvoir, 1949: 16).

Finalmente, las lógicas del orden de género se observan en uno de los ejercicios más "masculinos" del mundo social (y político): tomar la palabra. Vimos, en efecto, que los hombres y las mujeres del Concejo regional de Île-de-France no intervienen el mismo número de veces, ni acerca de los mismos temas, ni tampoco de la misma manera. En las sesiones de trabajo en comisiones, menos solemnes que las "sesiones plenarias", también se manifiesta la misma dominación masculina. Cualquiera sea su capital político, y al parecer contradiciendo los estereotipos más esperados, los hombres charlan mucho más con sus vecinos que las mujeres cuando otros miembros de la asamblea hacen uso de la palabra. Incluso algunos, más avezados, se levantan para hablar con un compañero sentado dos asientos más allá, lo que ninguna mujer en las dos comisiones hace (a menos que sea para ir a fumar un cigarrillo). Si añadimos que los hombres cortan mucho más la palabra que las mujeres y que también la toman antes de que se les haya dado, debemos constatar que los hombres, especialmente los que poseen más capital político, manifiestan algo como un derecho "natural" a expresarse en las comisiones. A la inversa, todo en el comportamiento de las mujeres (con mayor razón para las mujeres recién elegidas) manifiesta un sentimiento de ilegitimidad respecto a expresarse en este tipo de recinto. Primero, renuncian con mucha más facilidad que los hombres a tomar la palabra después de haberla pedido, con el pretexto de que un participante anterior ya habría dicho lo que querían decir. Luego, sus intervenciones son mucho más cortas que las de los hombres. E incluso si son más cortas, es porque las mujeres suelen hacer preguntas en vez de expresar una opinión. Cuando toman la palabra, buscan mucho más que los hombres obtener precisiones, informaciones e inclusive confirmaciones. A la inversa, los hombres, incluso cuando hacen preguntas, plantean muchas veces su opinión personal y/o su análisis político sobre el tema en discusión. Siempre hacen preceder su discurso de una pregunta, que en el fondo, afirma, recuerda y finalmente consolida ante todo su propia postura política. Cabe subra-

18. N.d.T.: Régimen parlamentario que tuvo Francia entre 1875 y 1940. 
yar finalmente que con un saber hacer y capitales equivalentes, las consejeras regionales difieren de los hombres en otro punto: "confiesan" mucho más fácilmente que ellos sus dudas, su ausencia de opinión e incluso su incompetencia. En otros términos, las mujeres expresan algo como un derecho "natural" a la incompetencia"

Como lo mostraron los sociolingüistas, lo que vemos aquí es en realidad la prolongación de lo que ocurre en el resto del mundo social. Pero puede ocurrir que la llamada al orden de género sea más apremiante que esta simple continuidad entre dos universos (que, por cierto, participa de la incorporación de las normas de dominación de los hombres). De este modo, cuando las mujeres intentan tomar la palabra de manera "masculina" (intervenciones en sesión plenaria, palabras muy generales, llamada al reglamento, polémica partidista...), la sanción es inmediata. Por ejemplo, Claire Le Flécher, del PS, durante una sesión dedicada a los "empleos trampolín" 20, medida estrella del mandato del presidente del Concejo regional socialista, atacó la política del gobierno e interpeló por su nombre al presidente de la bancada UMP. Uno de los consejeros generales llamó al orden, luego el presidente de la bancada UMP insistió, generando entonces desorden en la sesión bajo el pretexto de que Claire Le Flécher desconocería la regla según la cual no se ataca a las personas. (Cuando en realidad, este tipo de ataques ad hominem es frecuente, siendo el presidente de la bancada quien más recurre a ellos). El problema es el siguiente: renunciar a este tipo de forma de expresión, como por ejemplo a esta llamada al orden íntimo, significa también renunciar a hacerse notar y por lo tanto a perseguir una carrera partidista y política: la excelencia política incluye esta aptitud para intervenir respecto a todos los temas y en especial, para perderse en generalidades.

\section{Las esposas de políticos vs. las mujeres en política}

El ingreso de las mujeres en las arenas políticas llevó de manera paradójica a su reasignación a roles y actividades tradicionales. Esta reafirmación de los estereotipos puede también usar otros vectores más indirectos, pero igualmente eficientes. A partir de la campaña presidencial del 2002, el interés de los medios de comunicación y de los periodistas se desplazó hacia las esposas de los presidentes, como lo muestra Christiane Restier-Melleray. Sin embargo, la comunicación alrededor de esta figura es antigua en Francia: el Presidente (o el candidato a Presidente)

19. Cabe precisar, obviamente, que si bien el género es determinante en el hecho de tomar la palabra, poseer un capital político también lo es. De esta manera, un hombre elegido por primera vez manifiesta mucho menos facilidad que una mujer que tiene experiencia política.

20. N.d.T.: Los empleos trampolín corresponden a una subvención pagada por la región, que se entrega a empleadores que cumplen con una labor social. Esta subvención es una ayuda para la creación de contratos de trabajo permanentes. 
está casado y su esposa, aunque "excesivamente discreta, aparece en los relatos acerca de la arena pública" (Restier-Melleray, 1999: 90). Si bien no tiene existencia jurídica ni lugar en el protocolo, tiene, sin embargo, un papel de representación ("la dueña de casa, mi esposa", solía escribir Charles de Gaulle). Pero el examen de las revistas y de la prensa femenina desde la elección presidencial de 1965 muestra que la presencia de las esposas está creciendo. Por un lado, esto corresponde a una lógica política: la competencia entre candidatos para captar a las electoras aumenta, y las políticas públicas dirigidas a las mujeres se multiplican (contracepción, aborto, emancipación jurídica, etc.). Por otro lado, corresponde al lugar cada vez más amplio otorgado a la intimidad de los personajes públicos. En consecuencia, la primera función de la esposa del Presidente (o del candidato a Presidente) consiste en "mostrar físicamente la presencia del segundo sexo en el aparato estatal" (Restier-Melleray, 1999: 99). Este imperativo de representación las relega a los espacios internos y privados (la decoración, las obras sociales, la alta costura...). Son de esta manera transformadas en asistentes de sus maridos, incluso cuando son militantes como Danielle Mitterand o Sylviane Agacinski, la esposa de Lionel Jospin, candidato derrotado en 1995 y en 2002. Así, las mujeres cumplen también otra función que consiste en "autentificar las virtudes privadas de sus esposos", inclusive en restablecer la verdad acerca de ellos... (Restier-Melleray, 1999: 116).

Podríamos proseguir con este análisis al subrayar por una parte que este interés periodístico por las "Presidentas" se amplió recientemente a todas las esposas de ministros, en especial bajo el gobierno Raffarin ${ }^{21}$. Subrayamos, por otro lado, que todos los incumplimientos a los roles esperados también son sancionados, lo que constituye otra manera de reafirmar los estereotipos. Así ocurre por ejemplo con la divulgación de numerosas anécdotas acerca de Marie-Caroline Ferry, la esposa del ex ministro de Educación, Luc Ferry, como la siguiente, en la cual el tono, además, raya en la mofa:

En las cenas, no quiere parecer una mujer decorativa. No es su estilo. Le corta diez veces la palabra a su marido para exponer de manera más clara que él -piensa ella- la problemática de su tan complicado ministerio. Según un invitado habitual, "no siempre está on the point, como dicen los ingleses..." La joven esposa se queja: “Estoy harta de que me censuren! ¡Cuando pienso que me impediste dedicarme al cine!”. Luc Ferry subraya, incómodo: “iMira, querida, no podías aceptar un papel de prostituta antes de que entrara al gobierno!" 22

Esta reafirmación de los estereotipos es especialmente fuerte en el tratamiento periodístico dado a Bernadette Chirac, la esposa del actual jefe

21. N.d.T.: Primer Ministro entre el 2002 y el 2005, miembro de la mayoría presidencial UMP (Unión para un Movimiento Popular).

22. Chavelet (2003), p. 47. 
de Estado (Restier-Melleray, 2002). Si bien fue tratada durante mucho tiempo de la misma manera que las otras "Presidentas", ha sido objeto de un verdadero entusiasmo mediático a partir de 1997. Reelegida (consejera general de Corrèze) y con una posición cada vez más política (intervención contra el PACS, las cuotas, participación en la campaña municipal en la cual apoyó públicamente al candidato de la UMP en París, publicación de su libro Conversation en octubre del 2001), es presentada en forma progresiva como una mujer autónoma respecto de su marido. En el diario Le Figaro, la periodista Christine Clerc la presenta de la manera siguiente: "Ni demasiado joven, ni demasiado flaca, ni demasiado feminista... encarna los valores gaullistas del ayer, que quizá serán los valores del mañana" ${ }^{23}$. Este retrato permite diagnosticar la existencia de un verdadero backlash. Es como si, después de la paridad, nos enfrentásemos a un "proceso [que] obedece a una suerte de movimiento pendular, abriendo aparentemente la arena a la palabra de las mujeres, pero también al relato sobre las mujeres. Un relato que, hoy en día, es caracterizado por un encuadre novelesco del género femenino, propicio al retorno de una representación conservadora" (Restier-Melleray, 2002: 13). Bernadette Chirac se plantea a la vez como una mujer herida (por las infidelidades de su marido, que menciona entre líneas en su libro y en sus entrevistas) y como el elemento de una pareja en la cual existe una verdadera división sexual (y clásica) del trabajo. Igualmente, insiste en el lugar de la mujer como eje de la familia. Bernadette Chirac aparece entonces como una encarnación muy conservadora de las mujeres, frecuentemente puesta en escena en este período caracterizado a la vez por la aplicación de la paridad y de manera más general por controversias acerca de las problemáticas sexuales.

\section{Ségolène Royal: el estereotipo en pareja}

En esta configuración en la cual los estereotipos son reactivados como si fuesen llamadas al orden, ¿de qué manera la prensa y los profesionales de la política tratan a una mujer que pretende, a pesar de todo, ser candidata a la presidencia de la República ${ }^{24}$ ? Pregunta adicional: ¿qué tipo de mujer cabe ser hoy para "ser candidata a la candidatura"? ${ }^{25}$. Al respecto, el caso de Ségolène Royal es muy interesante. Nacida en 1953, esta mujer pertenece al grupo muy cerrado de las mujeres socialistas, ex alumnas de la Escuela Nacional de Administración (ENA), quienes han

23. Citado en Restier-Melleray (2002).

24. En la V República, el Presidente de la República posee varias e importantes prerrogativas, que puede ejercer de forma casi discrecional. Es por lo tanto la ubicación más codiciada y toda la vida política gira alrededor de esta posición, así como de su conquista.

25. N.d.T.: La expresión en francés destaca el momento en que los eventuales candidatos dan a conocer sus aspiraciones públicamente, antes de que su partido político tome una decisión al respecto. 
ocupado ministerios en los años 80 (Achin, 2005b). Fue ministra de Medio ambiente (1992-1993), ministra delegada a la Educación y luego a la Familia y la Infancia (1997-2002). Diputada desde 1988, es hoy la única mujer presidenta de un Concejo regional, el de Poitou-Charentes. Por otro lado, Ségolène Royal posee otra característica central, que cambia la manera en que los medios de comunicación y sus pares la tratan: ser, desde sus estudios compartidos en la ENA, la pareja de François Hollande, primer secretario del Partido Socialista, con quien tiene cuatro hijos.

Desde septiembre de 2005 y más aún desde el invierno del 2006, Ségolène Royal ha sido objeto de un fuerte entusiasmo mediático, a tal punto que fue portada de numerosos semanarios, desde los más políticos hasta los más populares. Se publicaron tres libros acerca de ella en menos de un año y obviamente se multiplicó el número de artículos sobre ella; esta producción debe mucho al aparato comunicacional que la rodea. Finalmente, mientras el 94\% de los franceses dice que apoya el hecho que una mujer sea Presidente de la República ${ }^{26}$, a partir del 2006, las empresas de sondeo incluyen su nombre en las encuestas para evaluar el apoyo electoral que recibiría en una segunda vuelta, y siempre le gana a su principal contendor de la derecha, el actual ministro del Interior, Nicolas Sarkozy.

Como en el caso de las demás mujeres, el cuerpo de Ségolène Royal es el objeto de las miradas. La prensa evoca sus tacos aguja -como ya se mencionó-, la cirugía estética que habría corregido su sonrisa e incluso los reparos de la marca con la cual se viste:

Cuesta creerlo, pero a la marca Paula Ka, que viste a las mujeres jóvenes y elegantes del distrito 16 de París, no le gusta demasiado que Ségolène Royal se exhiba en los escenarios de la televisión con dos de sus trajes sastre estrella: el azul marino con ribetes e incluso el beige... La dirección de la marca considera que Ségolène no corresponde para nada a su clientela: demasiado vieja y clásica. Pero ya que esta ropa es comprada y no prestada, la marca -que no es tampoco el último grito de la moda- debe aguantar en silencio esta "contrapublicidad" de Ségolène... (LePoint.fr, 15 de abril del 2006).

Al igual que otras representantes, Ségolène Royal es presentada como la "hija de". Su padre, ex coronel muy autoritario, machista y de extrema derecha, es el primer personaje que aparece en todas sus biografías y en los artículos acerca de ella. Aparece sistemáticamente como la persona en contra de quien se construyó, en sus opiniones políticas y feministas. Al igual que las otras mujeres, Ségolène Royal también es una esposa. En el 2003, en un libro dedicado a las esposas de los políticos, la periodista de Paris-Match, Elizabeth Chevalet, todavía la ubicaba en esta categoría y le daba un trato similar a la mujer de Nicolas Sarkozy en el mismo capítulo. Sin embargo, subrayaba lo siguiente:

26. Sondeo IFOP para Elle, 12-13 de enero del 2006. 
Cecilia Sarkozy se ve primera dama; Ségolène Royal está dispuesta a tomar ella misma el puesto si por casualidad le va mal a su pareja. No se echaría atrás ante el título de la primera mujer Presidenta de la República francesa (Chevalet, 2003: 15).

Desde entonces, otros libros solucionaron de otra manera el problema, al hablar de la pareja. Tanto el uno como el otro juegan con la ambigüedad: ¿quién será candidato? y por consecuente, ¿quién será la pareja del (de la) Presidente(a)? Si bien los candidatos tienen una esposa quien es su fiel valedor, en el caso de las mujeres, es como si las candidatas siempre tuvieran un esposo que mueve los hilos tras bastidores. Más aún, la candidatura de una mujer a la presidencia parece una cosa tan trasgresora que sólo se puede ver a esta mujer como parte de un matrimonio y, al final de cuentas, al matrimonio entero como candidato.

Finalmente (y sobre todo), Ségolène Royal es madre. Ya en 1992, Paris Match publicó fotografías de ella en la clínica, con su cuarto hijo recién nacido. Uno de los libros la apoda la "madona" y retoma este nombre en su título: La Madona y el Mono porfiado (el sobrenombre de su marido, François Hollande). Encontramos también este apodo bajo la pluma de Daniel Bernard, periodista del semanario Marianne, autor de Madame Royal. La misma expresión es retomada por Serge Raffy, editorialista de la revista económica de izquierda, Challenges. Éste reproduce además una cita de uno de sus pares, Jean-Christophe Cambadélis, citada por Daniel Bernard:

Es su foto preferida. Es del 17 de junio de 1988. Ségolène Royal posa, con un traje de chaqueta con flores, la mirada aureolada con una ternura infinita, la pupila en el eje de la cámara. Sostiene de manera relajada en sus brazos a su hijo Julien, un hermoso bebé de 6 meses, que toma concienzudamente su mamadera. En esta pareja, cada uno se dedica a sus cosas. El niño se alimenta. La madre nos atrae. Ségolène Royal se muestra. Está risueña, resplandeciente. La Señora ministra en campaña. No falta nada. Ni el menor detalle. Ni la jardinera Oshkosh del chiqui1lo, ni los aros con perlas de la mamá [...] "Es un Murillo, explica JeanChristophe Cambadélis, diputado del Partido Socialista de París. Es tan luminosa como la Muchacha con flores, sonriente como el rostro de la Virgen de la victoria de la catedral de Senlis ${ }^{27}$, pero nos mira como la Virgen en majestad de los Museos reales de Bruselas". ¿Senlis? ¿Bruselas? El icono ya está aquí. Y bien instalado. El icono de una guerrera que exhibe su orgullo, belleza y maternidad en un mismo impulso. Como telón de fondo, como un eco, se ve una forma de religiosidad tranquila. No hay vitrales, pero uno se los imagina. Ségolène Royal es una extraña mezcla de madona y de madre superiora. Su mensaje, desde hace más de veinte años, casi podría resumirse a través de una foto de una valkiria descansando ${ }^{28}$.

27. N.d.T.: En el tímpano de la catedral Nuestra Señora de Senlis, ciudad del norte de Francia, se ve una virgen coronada, recibiendo la bendición de Cristo.

28. Challenges, 13 de abril del 2006. 
Laurent Joffrin, jefe de redacción del Nouvel Observateur, una revista cercana al socialismo, extrae su descripción de un imaginario en parte más moderno, pero no obstante comparable:

Para algunos, es como Uma Thurman en la película Kill Bill, que corta en pedazos a sus adversarios con sablazos mediáticos. Para otros, es una especie de Santa Teresa de Lisieux ${ }^{29}$, quien suscita una devoción irracional $^{30}$.

En Madame Royal, Daniel Bernard amplía este registro de estereotipos al conjunto de la trayectoria de Ségolène Royal: "ingenua" (Bernard, 2005: 34), "madona con una cinta de terciopelo para el cabello" (Bernard, 2005: 90), "su perfil de medalla evoca la imaginería piadosa" (Bernard, 2005: 135). Ocurre lo mismo con el muy derechista Quotidien de Paris, en el cual se escribió en junio de 1992: "la mujer con el sentido común ñoño característico de una prima del campo un poco aguda, y con los modales amablemente altivos de una dama de caridad modernizada". Vale la pena profundizar estas comparaciones: la valkiria es una virgen guerrera; Juana de Arco es por supuesto la Doncella de Orleáns; una madre superiora o Teresa de Lisieux están condenadas a la castidad; el futuro marido de Kill Bill es exterminado durante la boda. La representación de Ségolène Royal (cuidadosamente puesta en escena, por el corte de pelo, el uso del color blanco, la sonrisa y las fotos "con el niño") contrasta con la imagen de las demás representantes. No ha sido reducida a su estatuto de objeto sexual: más aún, el deseo y la sexualidad le son denegados. El puesto presidencial está tan asociado a lo masculino, que introduce una verdadera "disputa en el género" 31 . Por otro lado, esta denegación tiene que ver con un fenómeno más general que abarca a todas las mujeres que se someten a la competencia política (Dulong, Matonti, 2005b): al generar demasiado, o al contrario, demasiado poco deseo, el cuerpo nunca es el que debería ser. ¿Cabe entonces suponer que la mejor manera de deshacerse de él es volviéndolo intocable?

Al final de cuentas, si la candidatura de Ségolène Royal está tan mediatizada, es porque encarna la reproducción en la revolución. En efecto, ocupó puestos femeninos (educación, medio ambiente, familia...), con el sentido doble que le hemos dado a este término. Luego, se planteó como la defensora de la familia, posición conservadora en comparación con su pertenencia partidista: lucha contra la pedofilia y la pornografía, reafirmación de la solidaridad familiar entre generaciones, prohibición del uso del colaless en los liceos y colegios... Esta dimensión de familia la llevó también a oponerse a la adopción de niños por las parejas homo-

29. N.d.T.: hermana carmelita francesa considerada como santa. Nacida en 1873, murió en 1897 de tuberculosis.

30. Nouvel Observateur, 6 de abril del 2006.

31. N.d.T.: referencia a la obra de Judith Butler, Gender Trouble (1990), traducida al castellano como El Género en Disputa (2001). 
sexuales, así como al matrimonio gay. En cuanto a su feminismo, es muy moderado: feminización de los nombres de profesiones y promoción de la píldora del día después para las menores de edad. Esto muestra hasta qué punto el éxito de la "candidata a la candidatura" puede ser comprendido como una llamada al orden de género, finalmente acarreada por la revolución paritaria.

\section{Conclusión}

En consecuencia, la ley francesa de paridad ha tenido efectos paradójicos. Primero, y obviamente, modificó de manera sustancial la composición de los Concejos regionales y municipales, y mostró una imagen más "mixta" de la política. Queda por verse si esta modificación, más allá de la "justicia" devuelta a las electoras, surtirá efectos respecto a las políticas públicas. Queda también por saber si el inicio de la profesionalización de las recién llegadas tendrá al final de cuentas efectos de renovación en el reclutamiento político. Sin embargo, algo está seguro: en segundo lugar, la ley reforzó los estereotipos de género. En efecto, éstos son mucho más parecidos a lo que los women studies observaron en los 70. Pero más aún, las argumentaciones a favor de la ley, por ejemplo respecto al carácter concreto de las mujeres, llevaron al reforzamiento de dichos estereotipos. Igualmente, la prensa o los profesionales de la comunicación contribuyeron a proponer modelos alternativos a los modelos de mujeres ligados a la política, influenciados por el "eterno femenino". Pero de nuevo queda por saber si esta inercia, inclusive este backlash, son una mera fase de resistencia antes de que la paridad se extienda a la esfera económica e incluso a la esfera doméstica.

\section{Bibliografía}

Achin, Catherine. 2005a. "Le mystère de la chambre basse". Comparaison des processus d'entrée des femmes au parlement, 1945-2000. Paris: Dalloz.

2005b. “Un métier d'hommes? Les représentations du métier de député à l'épreuve de sa féminisation". Revue Française de Science Politique 55 (3): 477-499.

Bailey, Frederick Georg. 1971. Les règles du jeu politique. Paris: PUF.

Beauvoir (de), Simone. 1949. Le Deuxième sexe. Paris: Gallimard.

Bereni, Laue. 2004. "Le mouvement français pour la parité et l'Europe". En Jacquot, Sophie, et al. (dir.), Les usages de l'Europe: Acteurs et transformations européennes. Paris: L'Harmattan, 33-54.

Bourdieu, Pierre. 1977. "Remarques provisoires sur la perception sociale du corps". Actes de la Recherche en Sciences Sociales 14: 51-54. 
Chavelet, Élisabeth. 2003. Leurs femmes... elles ont aussi du pouvoir. París: Robert Laffont.

Collovald, Annie. 1999. Jacques Chirac et le gaullisme. Biographie d'un héritier à histoires. París: Belin.

Cossart, Paula. 2001. "La Communion militante: les meetings de gauche durant les années Trente". Sociétés \& Représentations 12: 131-141.

Dulong, Delphine. 1997. Moderniser la politique. Aux origines de la Ve République. París: L'Harmattan.

2003. “Des actes d'institutions d'un genre particulier. Les conditions de légitimation des femmes sur la scène électorale (1945 et 2001)". En Lagroye, Jacques (dir.). La politisation. París: Belin, 425-443.

Dulong, Delphine; Lévêque, Sandrine. 2002. "Une ressource contingente. Les conditions de reconversion du genre en ressource politique". Politix 15 (60): 81-111.

Dulong, Delphine; Matonti, Frédérique. 2005a. "Entrer dans le rôle: le cas de nouvelles entrantes au Conseil régional". Communications aux Journées d'études "Linvention de l'élue". Bordeaux, 23-24 juin 2005.

2005b. “La mise en récit des femmes en campagne: l'indépassable féminité". En Lagroye, Jacques; Lehingue, Patrick; Sawicki, Frédéric (dir.). Mobilisations électorales. París: PUF, 279-303.

Dunezat, Xavier. 1998. "Des mouvements sociaux sexués". Nouvelles questions féministes, 19 (2-3-4): 161-195.

Fabius, Laurent. 1998. Les Blessures de la Vérité. París: Flammarion.

Fabre, Clarisse; Fassin, Éric. 2005. Liberté, égalité, sexualités. París: 10/18.

Freedman Jane. 1997. Femmes politiques. Mythes et symboles. París: L'Harmattan.

Garraud, Philippe. 1989. "Savoir faire et mobilisation des croyances dans le métier de maire". Politix 5: 11-16.

Gaxie, Daniel. 1997. "Économie des partis et rétributions du militantisme". Revue Française de Science politique 27 (1): 123-154.

Goffman, Erving. 1988. “La ritualisation de la féminité". En Les Moments et leurs hommes. París: Seuil / Minuit, 150-185.

Lagrave, Rose-Marie. 2000. “Une étrange défaite. La loi constitutionnelle sur la parité". Politix 13 (51): 113-141.

Latté, Stéphane. 2002. “Cuisine et dépendance. Les logiques pratiques du recrutement politique". Politix 15 (60): 55-80.

Le Grignou, Brigitte; Neveu, Érik. 1983. “Intimités publiques. Les dynamiques de la politique à la televisión". Revue Française de Science Politique 43 (6): 940-969.

Lévêque, Sandrine. 2005. “La féminité 'dépassée'? Usages et non-usages du genre dans les professions de foi des candidat(e)s parisien(ne)s aux élections législatives de 2002". Revue Française de Science politique 55 (3): 501-520.

Legavre, Jean-Baptiste. 2002. “'La queue du paon' ou les logiques de la production du portrait politique dans Libération". Sociétés \& Représentations: 375-391. 
Matonti, Frédérique (ed.). 2005La démobilisation politique. París: La Dispute.

Neveu, Érik. 1997. “Des questions 'jamais entendues'. Crise et renouvellements du journalisme politique à la télévision". Politix 37: 25-56.

Offerlé, Michel. 2002. Les partis politiques. París: PUF.

Rennes, Juliette. 2005. Le Mérite et la Nature. Une controverse républicaine: la mixité du prestige professionnel (1880-1990). Thèse de science politique, bajo la dirección de Pierre Birnbaum, Université Paris-I-Panthéon-Sorbonne.

Restier-Melleray, Christiane. 1999. “La femme du présidentiable. Une figurante engagée". En Mazet, Pierre; Poirmeur, Yves, Le métier politique en représentation. París: L'Harmattan: 89-159.

l'AFSP.

Sineau, Mariette. 1988. Des femmes en politique. París: Economica.

Troupel, Aurelia. 2002. “Disparités dans la parité. Les stratégies de contournement de la parité dans le département des Alpes-Maritimes". Politix 15 (60): 147-168. 